Original Paper http://ajol.info/index.php/ijbes http://indexmedicus.afro.who.int

\title{
Etude de la diversité, de l'endémisme et de la distribution spatiale des Rubiaceae de Côte d'Ivoire
}

\author{
Fatou Nanou TUO ${ }^{1}$, Kouao Jean KOFFI ${ }^{1 *}$, Akossoua Faustine KOUASSI ${ }^{2}$, \\ Moussa KONE ${ }^{1}$, Bakayoko ADAMA ${ }^{1}$ et Jan BOGAERT ${ }^{3}$ \\ ${ }^{1}$ Université Nangui Abrogoua, UFR-SN, 02 BP 801 Abidjan 02, Côte d'Ivoire. \\ ${ }^{2}$ Centre National de Floristique, 22 BP 582 Abidjan 22, Côte d'Ivoire. \\ ${ }^{3}$ Université de Liège, Gembloux Agro-Bio Tech Unité Biodiversité et Paysage, \\ Passage des Déportés, 2, B-5030 Gembloux, Belgique. \\ ${ }^{*}$ Auteur correspondant ; E-mail : kouaojean@yahoo.fr Tel : (+25) 47589663
}

\section{RESUME}

Cette étude a pour but de relever la diversité et l'endémisme des Rubiaceae, de faire une analyse de la distribution spatiale de ses espèces recensées en Côte d'Ivoire. Le Système d'Information Géographique (SIG) a été utilisé pour réaliser des cartes de distribution spatiale de chaque espèce. Le choix de la famille des Rubiaceae se justifie par le fait qu'elle est l'une des familles les plus importantes de la flore tropicale tant sur le plan floristique par l'abondance et la variété de ces espèces que sur le plan économique, médical et ornemental. Les échantillons utilisés pour cette étude ont été extrait de la base de données SIG IVOIRE. Le système de maillage a été utilisé et à travers les mailles, des indices de structure spatiale ont été calculés. L'aspect floristique a révélé 1623 échantillons regroupés en 83 genres et 282 espèces dont $2 \%$ de ces espèces sont endémiques à la Côte d'Ivoire. La carte de distribution spatiale de l'ensemble des échantillons a mis en évidence les degrés de prospection sur le territoire ivoirien.

(C) 2017 International Formulae Group. All rights reserved.

Mots clés: Distribution spatiale, diversité, endémisme, Rubiaceae, Côte d'Ivoire.

\section{Study of the diversity, endemism and spatial distribution of the Rubiaceae of Côte d'Ivoire}

\begin{abstract}
This study aimed at identifing the diversity and endemism of the Rubiaceae, analyzing the spatial distribution of species found in Côte d'Ivoire. The Geographic Information System (GIS) was used to produce spatial distribution maps of each species. The choice of the Rubiaceae family is justified by the fact that it is one of the most important families of the tropical flora both in terms of floristics by the abundance and variety of these species and economic, medical and ornamental.The samples used were extracted from the SIG IVOIRE database. A grid system was used to cover the Ivorian territory, Spatial structure indices were calculated. The floristic aspect revealed 1623 samples grouped into 83 genera and 282 species of which $2 \%$ are
\end{abstract}


endemic to Côte d'Ivoire. The spatial distribution map of all the samples showed the degrees of prospecting in Côte d'Ivoire. Some areas were more explored than others.

(C) 2017 International Formulae Group. All rights reserved.

Keywords: Spatial distribution, Diversity, endemism, Rubiaceae, Côte d'Ivoire.

\section{INTRODUCTION}

Les investigations botaniques en Côte d'Ivoire, pays de l'Afrique de l'ouest, datent des années 1882 et ont été réalisées par Chaper. Cependant, c'est Auguste Chevalier qui fut celui qui a commencé l'étude floristique de la Côte d'Ivoire (Aké Assi, 2001, 2002). Ces investigations floristiques ont été poursuivies, surtout en région guinéocongolaise, durant les précedentes décennies. En 1900, le territoire ivoirien possédait 16 millions d'hectares de forêt naturelle dense humide (Koné et al., 2014). La Côte d'Ivoire a connu un recul de ses surfaces forestières (Mayaux et al., 2003; Brou et al., 2005 ; Koné et al., 2014). Le couvert forestier ivoirien, estimé à environ 3 millions d'hectares, par une étude en 1988, est passé à moins de 2 millions d'hectares aujourd'hui (Koné et al., 2014). Ainsi, les forêts ivoiriennes n'échappent pas au phénomène de déforestation que connaît la majorité des forêts d'Afrique (Chatelain et al., 2004 ; Assoumou et al., 2012). La politique de développement de la Côte d'Ivoire basée sur l'exploitation forestière et sur l'agriculture a entraîné une régression drastique de ses surfaces forestières ainsi qu'une modification profonde de son milieu naturel. Cette dégradation galopante $\mathrm{du}$ milieu naturel ivoirien avec ses ressources biologiques est aussi due à la forte pression démographique que connaît le pays. Ce phénomène a malheureusement provoqué la disparition de milliers d'hectares de forêts avec pour conséquence l'extinction de nombreuses espèces végétales (Menziès, 2000) dont celles de la famille des Rubiaceae. Les Rubiaceae sont des plantes très riches en espèces (Davis et Figueiredo, 2007 ; Lachenaud et al., 2013 ; Lachenaud, 2013) avec un nombre estimé, au niveau mondial à 13143 espèces regroupées en 611 genres environ (Davis et al., 2009). En Côte d'Ivoire, cette famille, très importante, comme certaines familles botaniques telles que les Asteraceae, les Leguminoseae (Eddouks et al., 2007; Lebri et al., 2015 ; Ambé, 2015; Yapi, 2015), est utilisée dans plusieurs domaines de la vie courante tels que l'alimentation, la santé. De plus, les espèces servent aussi à divers autres usages (Aké Assi, 2001, 2002; Kouassi et al., 2015). Face à l'ampleur de la déforestation de notre milieu naturel et vu le rôle des Rubiceae dans la vie des populations ivoiriennes, la gestion durable de nos ressources forestières s'avère indispensable. Cette étude est alors une contribution à la connaissance et à la distribution des espèces de la famille des Rubiaceae de Côte d'Ivoire après le constat de la dégradation avancée de nos forêts. De façon spécifique, il s'agira de relever la diversité, l'endémisme des Rubiaceae et de faire une analyse de la distribution spatiale de ses espèces recensées en Côte d'Ivoire.

\section{MATERIL ET METHODES}

\section{Collecte des données}

Pour la collecte des données, la base de données SIG IVOIRE (Chatelain et al., 2001) qui comporte 1623 échantillons de Rubiaceae a été consultée. Le site «theplantlist.org»a 
servie pour la vérification des noms scientifiques des espèces. Les données recueillies ont été triées et corrigées, de même que les noms et les coordonnées géographiques ont été vérifiés. La compilation des espèces de Rubiaceae de la liste corrigée a permis d'avoir un nombre d'occurrence des espèces recensées. Les espèces les plus récoltées ont été identifiées. Une analyse floristique a été effectuée afin de répertorier les différents types biologiques et chorologiques des espèces de Rubiaceae en se référant à la flore de Côte d'Ivoire (Aké Assi, 2001, 2002) et d'estimer la diversité floristique. Le coefficient $\mathrm{E} / \mathrm{G}$ (rapport $\mathrm{du}$ nombre d'espèces sur le nombre de genres) a été utilisé pour estimer la diversité floristique. Une faible valeur (proche de 1) de ce coefficient témoigne de la forte diversité de la flore.

\section{Traitement des données}

Pour cette étude, un système de maillage a été utilisé, méthode utilisée par plusieurs auteurs tels que Crisp et al. (2001) et Koffi (2008). C'est un processus avec une démarche analytique par l'utilisation des mailles de différentes tailles : $0,25^{\circ}-0,50^{\circ}$ $0,75^{\circ}-1,00^{\circ}-1,25^{\circ}-1,50^{\circ}-1,75^{\circ}$. Ces analyses ont pour but de choisir la taille qui permet d'avoir au moins $10 \%$ de mailles vides afin d'éviter la perte énorme d'informations comme indiquée sur la Figure 1, c'est la maille de taille $1^{\circ} \times 1^{\circ}$ qui permet d'avoir moins de $10 \%$ de mailles vides. Dans la suite du travail, des mailles de $1^{\circ} \times 1^{\circ}$ numérotées de 0 à 43 , sont retenues pour le calcul des différents indices d'analyse spatiale (Figure 2).

La détermination de la valeur de la Richesse spécifique estimée $\left(S_{e s t}\right)$ se fait par utilisation de l'estimateur de premier ordre de jackknife (Burnham et Overton, 1978). Il a été calculé avec la version 9.1.0 du logiciel
Estimate $\mathrm{S}$. La richesse spécifique observée $\left(S_{\text {obs }}\right)$ et la richesse spécifique estimée $\left(S_{\text {est }}\right)$ permettent la réalisation de la courbe d'accumulation. Le rapport de Richesse spécifique observée et la Richesse spécifique estimée permet de calculer le taux de l'effort d'échantillonnage par la formule suivante :

$$
\text { Effort d'échantillonnage }=\frac{\text { Sobs }}{\text { Sest }} \times 100
$$

La diversité maximale est donnée par la formule de Shannon et Weaver (1949) et est noté $H^{\prime}$. $H^{\prime}$ a été calculé avec le logiciel Estimate $\mathrm{S}$ par la formule :

$$
H^{\prime}=\sum(P i) \log 2(P i)
$$

avec : $H^{\prime}=$ Indice de diversité de Shannon, $P_{i}$ $=$ Proportion de l'espèce i, $S=$ Nombre total d'espèces. $H$ ' s'exprime en bits par individus et peut prendre la valeur entre 0 et $\log 2(S)$.

Aussi l'indice d'équitabilité ou régularité de Pielou (1975) a été calculé à travers les mailles, par la fraction ci-dessous:

$$
E=-\frac{\sum_{i=1}^{n} P i \log P i}{\log n}
$$

Avec $p_{i=}$ la proportion des mailles de chaque zone, $n=$ le nombre de subdivisions. $E$ est une valeur comprise entre 0 et 1 . La distribution spatiale des espèces est équitable lorsque $E=$ 1.

Quant à l'indice de dispersion, il permet de caractériser le modèle spatiale. Sa formule est :

$X^{2}=\sum_{i=1}^{m}(n i-\mu)^{2} / \mu, \quad$ Avec $\mu=\sum n i / m$, $n i=$ la fréquence proportionnelle de chaque espèces, $n=$ le nombre de subdivisions, $m=1 \mathrm{e}$ nombre d'échantillon contenu dans toute les mailles, $\mu=$ la moyenne. Une valeur élevée de $X^{2}$ indique une distribution spatiale agrégative des espèces, tandis qu'une faible valeur de $X^{2}$ montre une régularité de la distribution des espèces du milieu.

A l'aide du logiciel cartographique QGIS version 2.6 (http: //www.qgis.org), des cartes de distribution spatiale des espèces de Rubiaceae ont été réalisées. 


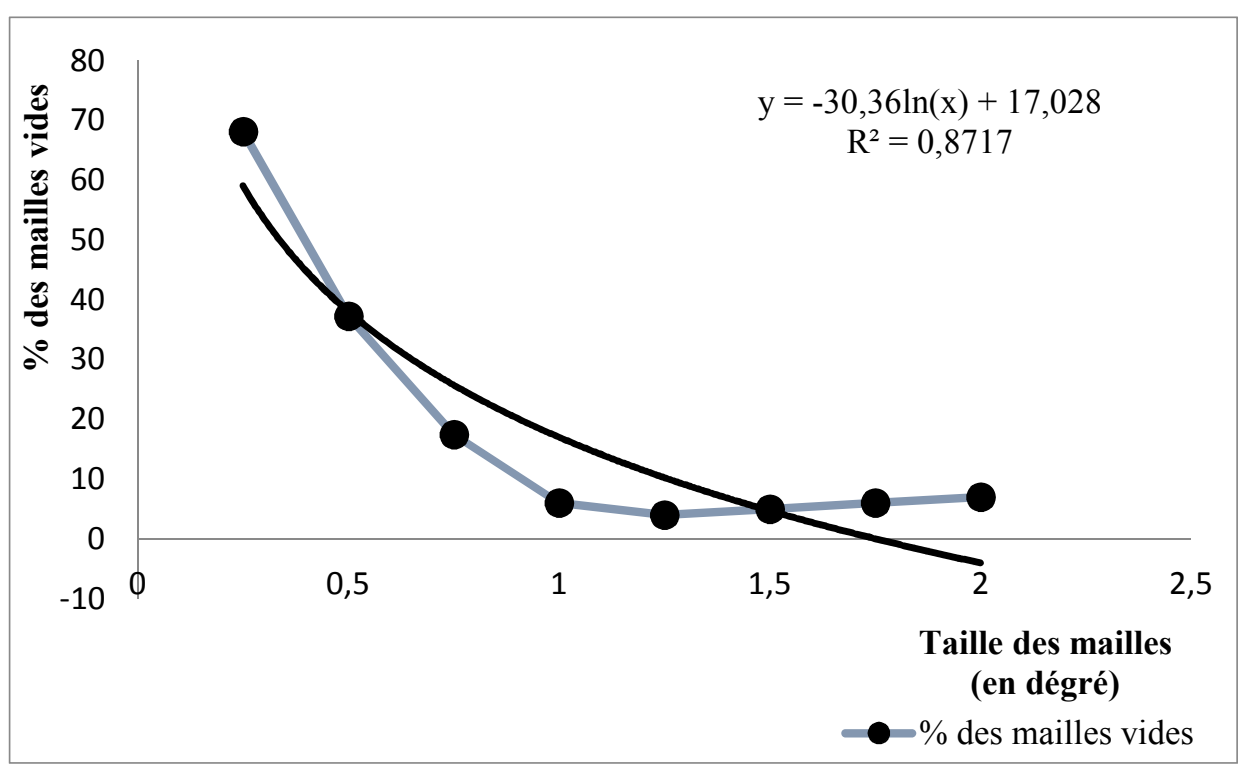

Figure 1 : Taille des mailles en fonction du pourcentage (\%) des mailles vides.

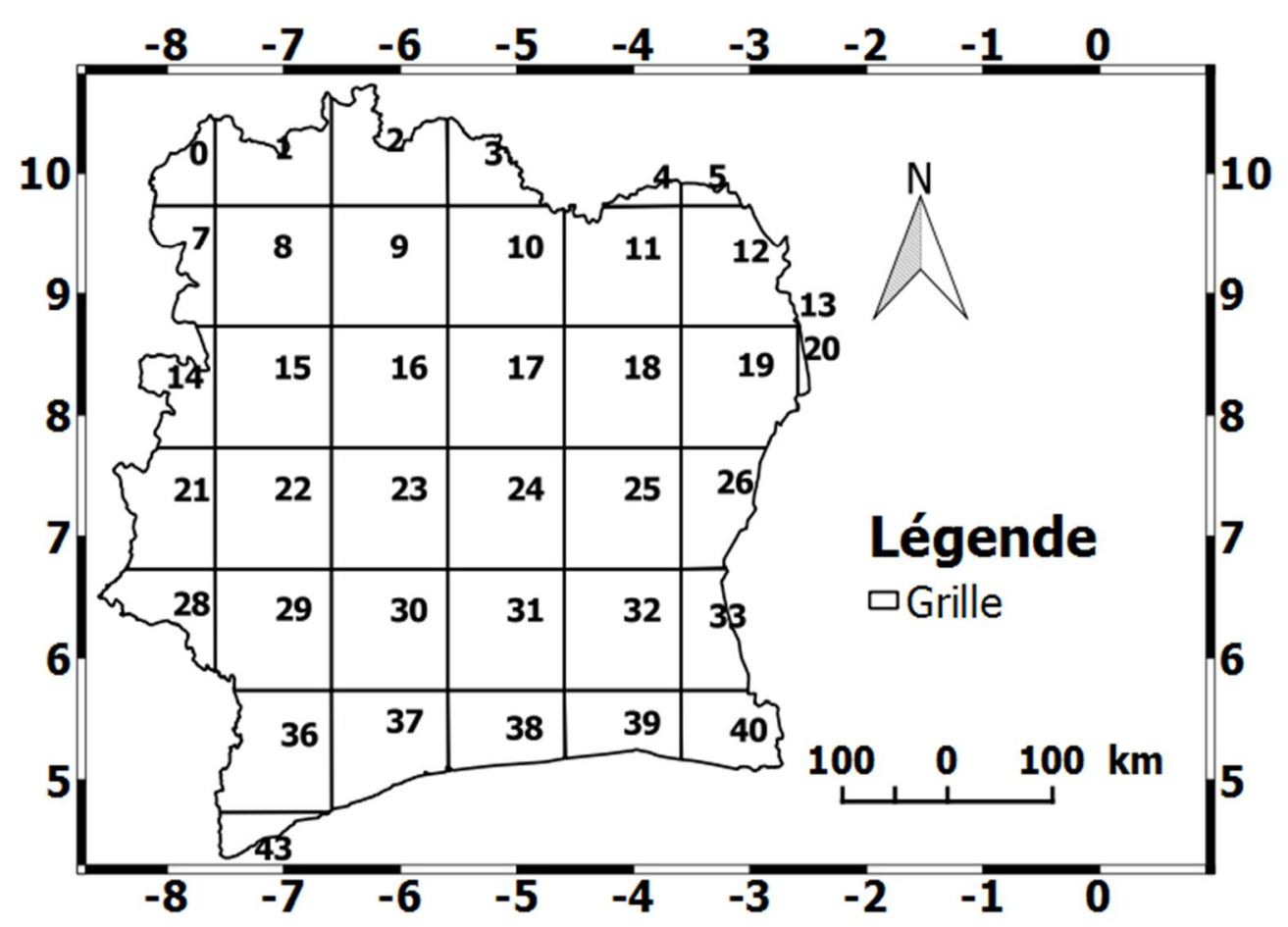

Figure 2 : Représentation de mailles de $1^{\circ} \times 1^{\circ}$ numérotées de 0 à 43 sur toute 1'étendue la Côte d'Ivoire. 


\section{RESULTATS}

Diversité floristique et endémisme de la famille des Rubiaceae

Les travaux effectués ont permis de dresser une liste de 1623 échantillons, regroupés en 83 genres et 282 espèces (Tableau 1) issus de 298 localités. Les genres qui regroupent les plus grands nombre d'espèces sont : Psychotria L. (39 espèces), Spermacoce (15 espèces), Keetia (11 espèces). Par contre les genres les moins représentés sont: Vahlia, Oligocodon, Pausinystalia, Polysphaeria, Hazaliella avec chacun une espèce. Les espèces les plus échantillonnées sont: Ixora brachypoda DC. (23 échantillons), Chassalia kolly (Schumach.) Hepper (23 échantillons), Sarcocephalus latifolius (Sm.) E.A. Bruce (22 échantillons). Par contre les espèces les moins récoltées sont: Ixora baldwinii Keay, Vangueriella glabrescens (Robyns) Verdc. avec 1 échantillon chacune. La base de données de la famille des Rubiaceae indique que $50 \%$ de ces espèces sont Guinéo-Congolaise. Les espèces endémiques du bloc forestier à l'Ouest du Togo, comprenant le Ghana, la Côte d'Ivoire, le Liberia, la Sierra Leone, la Guinée Bissau, la Guinée, la Gambie et le Sénégal et les espèces Guinéo-Congolaises-SoudanoZambéziennes représentent respectivement $23 \%$ et $19 \%$ (Figure 3 ). Il faut souligner que dans cette base de données, $2 \%$ des espèces sont endémiques de la Côte d'Ivoire. Il s'agit de Psychotria abouabouensis (Schnell) Verdc., Psychotria brachyanthoides De Wild., Psychotria peduncularis var. tabouensis (Schnell) Verdc. Sericanthe toupetou (Aubrév. \& Pellegr.) Robbr et Empogona reflexa var. ivorensis (Robbr.) Tosh \& Robbr. Concernant les types biologiques des espèces de la famille des Rubiaceae de la base de données, les microphanérophytes représentent $34 \%$, suivi des nanophanérophytes $26 \%$, des microphanérophytes lianescentes 18\%. Les mégaphanérophytes, les nanophanérophytes lianescentes et les hémicryptophytes pyrophytique ne représentent que chacun $1 \%$ des espèces (Figure 4). Le coefficient générique de la famille des Rubiaceae est donné par le nombre d'espèces sur celui du genre; il est de 0,29 soit $29 \%$.

\section{Analyse de distribution spatiale des Rubiaceae}

La carte de distribution de tous les échantillons montre une exploration irrégulière dans les différentes mailles. Certaines mailles telles que les mailles 21, 29, 31 et 39 sont plus compactes donc plus explorées, par contre, d'autre telles les mailles $17,25,36$, etc. le sont partiellement. La maille 0 n'a pas du tout été explorée (Figure 5). Ainsi la maille 21 correspond à la région du Tompki et à des parties du Guemon et du Cavally. La maille 29 rassemble les régions du Cavally, du Nawa et à des parties du Haut Sassandra et du Guemon. La maille 31 regroupe les régions de l'Agnéby-Tiassa, du Belier et une partie du Goh, de Loh-Djiboua, de Yamoussoukro, du Moronou, du N'Zi, de la Marahoué. La maille 39 quant à elle regroupe les régions d'Abidjan, de l'AgnébyTiassa en plus d'une partie de la Mé, du Sud Comoé et des Grands Ponts (Figure 6).

La représentation de la courbe d'accumulation des espèces dans les différentes mailles indique un taux de couverture de $79,05 \%$ qui est l'évolution globale de la richesse spécifique observée ( $S_{o b s}$ Mean) et de la richesse spécifique estimée $\left(S_{\text {est }}\right)$. La Figure 7 montre que cette évolution du nombre d'espèces augmente au 
fur et à mesure qu'on échantillonne dans les différentes mailles jusqu'à atteindre une limite qui est le plateau où le nombre d'espèces n'augmente pratiquement plus, ceci indique que les échantillons sont représentatifs. L'indice d'équitabilité $E=0.68$, calculé à travers les mailles, montre une irrégularité d'exploration à travers la Côte d'Ivoire. De plus, la valeur de l'indice de dispersion $\left(X^{2}=\right.$ 4.00278762; $\left.\quad X^{2}{ }_{\mathrm{m}-1}=59.3\right)$ montre une agrégation considérable.

Les différentes cartes de distribution spatiale des espèces mettent en évidence les différents patterns de distribution des espèces à travers les mailles: espèces à large distribution et espèces à distribution restreinte. Dans cette étude, une espèce à large distribution est une espèce récoltée dans plus d'une maille, par contre une espèce à distribution restreinte est considérée comme une espèce récoltée dans seulement une maille. Ainsi, dans la base de données SIG IVOIRE, il y a quelques espèces de Rubiaceae à large distribution telles que Rothmannia longiflora Salisb, avec 15 échantillons, récoltés dans 10 mailles (Figure 8) et Chassalia kolly (Schumach.) Hepper, avec 23 échantillons, récoltés dans 9 mailles (Figure 9). On a aussi quelques espèces à distribution restreinte telles que Psychotria limba Scott-Elliot, avec 4 échantillons (Figure 10) et Tricalysia reticulata (Benth.) Hiern, avec 7 échantillons (Figure 11) récoltés dans une seule maille.

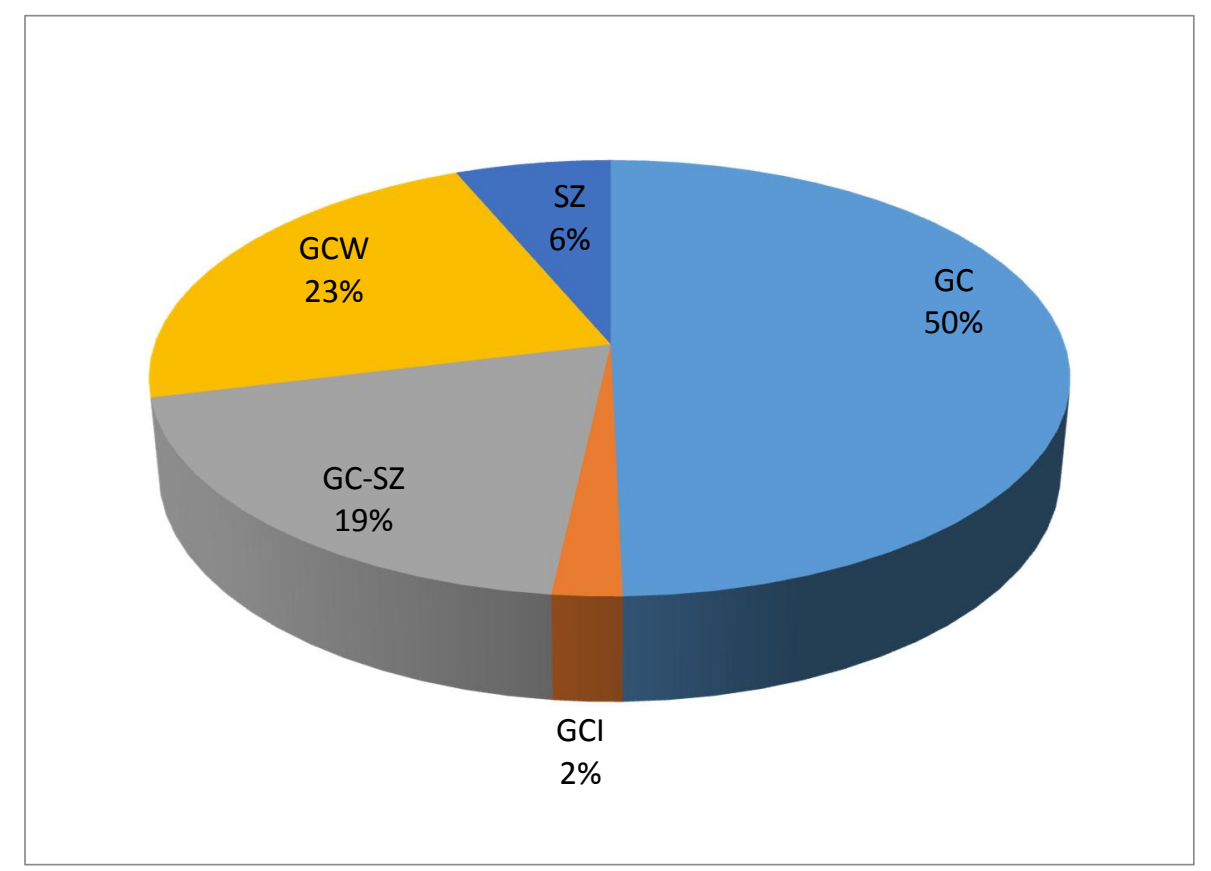

Figure 3 : Type chorologique des espèces de la famille de Rubiaceae de la base de données SIG IVOIRE (GC : Guinéo-Congolais ; GC-SZ : Guinéo-Congolaises-Soudano-Zambéziennes ; SZ : Soudano-Zambéziennes; GCW : espèces endémiques du bloc forestier à l'Ouest du Togo; GCI : espèces endémiques de la Côte d'Ivoire). 
Tableau 1: Liste des espèces de Rubiaceae.

\begin{tabular}{|c|c|c|c|}
\hline Nombres & Espèces & Chorologie & Type biologique \\
\hline 1 & Aidia genipiflora (DC.) Dandy & $\mathrm{GC}$ & $\mathrm{mp}$ \\
\hline 2 & Argocoffeopsis afzelii (Hiern) Robbr. & $\mathrm{GC}$ & Lmp \\
\hline 3 & Argocoffeopsis eketensis (Wernham) Robbr & GC-SZ & Lmp \\
\hline 4 & Argocoffeopsis rupestris (Hiern) Robbr. & GC & np \\
\hline 5 & Argostemma pumilum Benn. & GCW & Th \\
\hline 6 & Atractogyne bracteata (Wernham) Hutch. \& Dalziel & GC & Lmp \\
\hline 7 & Aulacocalyx jasminiflora Hook.f & $\mathrm{GC}$ & $\mathrm{mp}$ \\
\hline & Belonophora coffeoides Hook.f. subsp. hypoglauca (Welw. & & \\
\hline 8 & ex Hiern) S.E.Dawson \& Cheek & $\mathrm{GC}$ & $\mathrm{mp}$ \\
\hline 9 & Bertiera bracteolata Hiern & GC & Lmp \\
\hline 10 & Bertiera breviflora Hiern & $\mathrm{GC}$ & $\mathrm{np}$ \\
\hline 11 & Bertiera chevalieri Hutch. \& Dalziel & GCW & Lmp \\
\hline 12 & Bertiera fimbriata (A.Chev. ex Hutch. \& Dalziel) Hepper & GCW & $\mathrm{np}$ \\
\hline 13 & Bertiera racemosa (G.Don) K.Schum. & GC & $\mathrm{mp}$ \\
\hline 14 & Breonadia salicina (Vahl) Hepper \& J.R.I.Wood & SZ & $\mathrm{mp}$ \\
\hline 15 & Calycosiphonia spathicalyx (K.Schum.) Robbr & GC & Lmp \\
\hline 16 & Chassalia afzelii (Hiern) K.Schum & GCW & Lmp \\
\hline 17 & Chassalia corallifera (A.Chev. ex De Wild.) Hepper & GCW & np \\
\hline 18 & Chassalia elongata Hutch. \& Dalziel & GCW & $\mathrm{np}$ \\
\hline 19 & Chassalia kolly (Schumach.) Hepper & GC & np \\
\hline 20 & Chassalia laxiflora Benth. & GCW & np \\
\hline 21 & Chassalia subherbacea (Hiern) Hepper & $\mathrm{GC}$ & $\mathrm{Ch}$ \\
\hline 22 & Chazaliella lophoclada (Hiern) E.M.A.Petit \& Verdc. & GCW & $\mathrm{np}$ \\
\hline 23 & Chazaliella sciadephora (Hiern) E.M.A.Petit \& Verdc. & $\mathrm{GC}$ & $\mathrm{np}$ \\
\hline 24 & Coffea $\times$ arabusta Capot $\&$ Aké Assi & GC & $\mathrm{mp}$ \\
\hline 25 & Coffea canephoraPierre ex A.Froehner & GC & $\mathrm{mp}$ \\
\hline 26 & Coffea ebracteolata (Hiern) Brenan & GC & $\mathrm{np}$ \\
\hline 27 & Coffea humilis A.Chev & GCW & $\mathrm{np}$ \\
\hline 28 & Coffea liberica Hiern & $\mathrm{GC}$ & $\mathrm{mp}$ \\
\hline 29 & Coffea mannii (Hook.f.) A.P.Davis & GC & $\mathrm{np}$ \\
\hline 30 & Coffea stenophylla G.Don & GCW & $\mathrm{mp}$ \\
\hline 31 & Corynanthe pachyceras K.Schum & GC & $\mathrm{mP}$ \\
\hline 32 & Craterispermum caudatum Hutch & $\mathrm{GC}$ & $\mathrm{mp}$ \\
\hline 33 & Craterispermum cerinanthum Hiern & GC & $\mathrm{mp}$ \\
\hline 34 & Craterispermum laurinum (Poir.) Benth & GC & $\mathrm{mp}$ \\
\hline 35 & Cremaspora triflora (Thonn.) K.Schum. & GC-SZ & Lmp \\
\hline 36 & Crossopteryx febrifuga (Afzel. ex G.Don) Benth & GC-SZ & $\mathrm{mp}$ \\
\hline 37 & Cuviera acutiflora $\mathrm{DC}$ & GC & $\mathrm{mp}$ \\
\hline 38 & Cuviera macroura K.Schum. & GC-SZ & $\mathrm{mp}$ \\
\hline 39 & Cuviera nigrescens (Elliott ex Oliv.) Wernham & $\mathrm{GC}$ & $\mathrm{mp}$ \\
\hline
\end{tabular}


Dictyandra arborescens Welw. ex Hook.f

GC

mp

Didymosalpinx abbeokutae (Hiern) Keay

GC

Lmp

Diodella sarmentosa (Sw.) Bacigalupo \& Cabral ex

Borhidi

Diodella serrulata (P.Beauv.) Borhidi.

GC-SZ

Lnp

Diodia rubricosa Hiern

$\mathrm{GC}$

$\mathrm{Ch}$

Diodella scandens (Sw.) Bacigalupo \& E.L.Cabral

GC

$\mathrm{Ch}$

Empogona bracteata (Hiern) Tosh \& Robbr.

GC-SZ

Lnp

Empogona deightonii (Brenan) Tosh \& Robbr.

Empogona filiformistipulata (De Wild.) Bremek.

GCW

mp

GCW

np

Empogona macrophylla (K.Schum.) Tosh \& Robbr.

$\mathrm{GC}$

$\mathrm{Np}$

Empogona reflexa (Hutch.) Tosh \& Robbr.

GC

mp

Empogona reflexa var. ivorensis (Robbr.) Tosh \& Robbr.

$\mathrm{GC}$

$\mathrm{mp}$

Euclinia longiflora Salisb

$\mathrm{GCI} \quad \mathrm{mp}$

$\mathrm{GC} \quad \mathrm{mp}$

Fadogia cienkowskii Schweinf

$\mathrm{SZ}$

np

Fadogia erythrophloea (K.Schum. \& K.Krause) Hutch. \&

$\mathrm{SZ} \quad \mathrm{mp}$

SZ

mp

Feretia apodanthera Delile

GC

MP

Fleroya stipulosa (DC.) Y.F.Deng

GC-SZ

$\mathrm{mP}$

Gaertnera aurea Malcomber

Gaertnera cooperi Hutch. \& M.B.Moss

GCW

$\mathrm{mp}$

Gaertnera longivaginalis (Schweinf. ex Hiern)

GCW $\quad \mathrm{mp}$

\section{E.M.A.Petit}

GC

np

Gaertnera paniculata Benth

$\mathrm{GC}$

Gardenia aqualla Stapf \& Hutch

SZ

Gardenia erubescens Stapf \& Hutch

SZ

Gardenia imperialis K.Schum

GC-SZ

Gardenia nitida Hook

Gardenia ternifolia Schumach. \& Thonn

GC-SZ

Gardenia vogelii Hook.f.

Geophila afzelii Hiern

GC

np

Geophila obvallata Didr

GC

np

Geophila repens (L.) I.M.Johnst

$\mathrm{GC}$

GC-SZ

$\mathrm{mp}$

Hazaliella sciadephora (Hiern) E.M.A.Petit \& Verdc

GC

Heinsia crinita (Afzel.) G.Taylor

GC

GCW

Hutchinsonia barbata Robyns

GC

$\mathrm{Ch}$

Hymenocoleus hirsutus (Benth.) Robbr.

$$
\mathrm{GC}
$$

Hymenocoleus libericus (A.Chev. ex Hutch. \& Dalziel)

Robbr

GC

$\mathrm{Ch}$

Hymenocoleus multinervis Robbr

GCW

$\mathrm{Ch}$

Hymenocoleus nervopilosus Robbr.

GC

$\mathrm{Ch}$

Hymenocoleus rotundifolius (A.Chev. ex Hepper) Robbr

$\mathrm{GC}$

$\mathrm{Ch}$

Hymenodictyon floribundum (Hochst. \& Steud.) B.L.Rob

GC-SZ

mp 
Ixora aggregata Hutch.

Ixora baldwinii Keay

Ixora brachypoda DC

Ixora nigerica Keay

Ixora hiernii Scott-Elliot

Ixora laxiflora $\mathrm{Sm}$.

Ixora nimbana Schnell

Keetia bridsoniae Jongkind

Keetia cornelia (Cham. \& Schltdl.) Bridson.

Keetia hispida (Benth.) Bridson

Keetia leucantha (K.Krause) Bridson

Keetia mannii (Hiern) Bridson

Keetia multiflora (Schumach. \& Thonn.) Bridson

Keetia rubens (Hiern) Bridson

Keetia rufivillosa (Robyns ex Hutch. \& Dalziel) Bridson

Keetia tenuiflora (Welw. ex Hiern) Bridson

Keetia venosa (Oliv.) Bridson

Keetia venosissima (Hutch. \& Dalziel) Bridson

Kohautia confusa (Hutch. \& Dalziel) Bremek

Kohautia tenuis (Bowdich) Mabb

Lasianthus batangensis K.Schum

Leptactina densiflora Hook.f.

Macrosphyra longistyla (DC.) Hiern

Massularia acuminata (G.Don) Bullock ex Hoyle

Mitracarpus hirtus (L.) DC

Mitragyna inermis (Willd.) Kuntze

Morelia senegalensis A.Rich. ex DC

Morinda chrysorhiza (Thonn.) DC

Morinda longiflora $\mathrm{G}$. Don

Morinda lucida Benth

Morinda morindoides (Baker) Milne-Redh

Multidentia pobeguinii (Hutch. \& Dalziel) Bridson

Mussaenda afzelii G. Don

Mussaenda arcuata Poir.

Mussaenda chippii Wernham

Mussaenda elegans Schumach. \& Thonn

Mussaenda erythrophylla Schumach. \& Thonn

Mussaenda grandiflora Benth

Mussaenda isertiana DC.

Mussaenda nivea A.Chev. ex Hutch. \& Dalziel

Mussaenda tristigmatica Cummins

Nauclea diderrichii (De Wild.) Merr

Nauclea gilletii (De Wild.) Merr

Nauclea pobeguinii (Pobég. ex Pellegr.) Merr. ex E.M.A.
GCW

GCW

GC-SZ

np

mp

$\mathrm{mp}$

$\mathrm{GC} \quad \mathrm{np}$

$\mathrm{GC} \quad \mathrm{mp}$

GCW np

GCW L

SZ Lmp

GC Lmp

GC Lmp

GC Lmp

GC Lmp

GCW Lmp

GC LmP

GC Lmp

GC-SZ Lmp

GC Lmp

GC-SZ Hpy

GC-SZ Hpy

GC np

GC Lmp

GC-SZ Lmp (mp)

$\mathrm{GC} \quad \mathrm{mp}$

GC-SZ Th

$\mathrm{SZ} \quad \mathrm{mp}$

GC-SZ $\quad \mathrm{mp}$

GCW $\mathrm{mp}$

GC-SZ Lmp

GC-SZ $\mathrm{mp}$

GC Lmp

GCW mp

GC Lmp

GC-SZ Lmp

GCW Lmp

GC Lmp

GC Lmp

GCW Lmp

GC Lmp

GCW Lmp

GCW Lmp

GC MP

GC MP

GC-SZ $\mathrm{mP}$ 
Petit

Nauclea xanthoxylon (A.Chev.) Aubrév

Nichallea soyauxii (Hiern) Bridson

Oldenlandia affinis (Roem. \& Schult.) DC

Oldenlandia chevalieri Pit.

Oldenlandia corymbosa L.

Oldenlandia goreensis (DC.) Summerh

Oldenlandia herbacea (L.) Roxb

Oldenlandia lancifolia (Schumach.) DC

Oldenlandia wauensis Schweinf. ex Hiern

Oligocodon cunliffeae (Wernham) Keay

Otomeria cameronica (Bremek.) Hepper

Otomeria elatior (A.Rich.) Verdc.

Oxyanthus formosus Hook.f.

Oxyanthus pallidus Hiern

Oxyanthus racemosus (Schumach. \& Thonn.) Keay

Oxyanthus speciosus DC

Oxyanthus subpunctatus (Hiern) Keay

Oxyanthus unilocularis Hiern

Parapentas setigera (Hiern) Verdc

Pauridiantha afzelii (Hiern) Bremek

Pauridiantha hirtella (Benth.) Bremek

Pauridiantha stipulosa (Hutch. \& Dalziel) Hepper

Pauridiantha ziamaeana (Jacq.-Fél.) Hepper

Pausinystalia lane-poolei (Hutch.) Hutch. ex Lane-Poole

Pavetta corymbosa (DC.) F.N.Williams

Pavetta crassipes K.Schum.

Pavetta ixorifolia Bremek

Pavetta lasioclada (K.Krause) Mildbr. ex Bremek

Pavetta micheliana J.-G.Adam

Pavetta mollissima Hutch. \& Dalziel

Pavetta oblongifolia (Hiern) Bremek

Pavetta owariensis P.Beauv.

Pavetta platycalyx Bremek

Pentodon pentandrus (Schumach. \& Thonn.) Vatke

Pleiocoryne fernandensis (Hiern) Rauschert

Polysphaeria arbuscula K.Schum

Pouchetia africana A.Rich. ex DC.

Psychotria abouabouensis (Schnell) Verdc.

Psychotria adafoana K.Schum

Psychotria albicaulis Scott-Elliot

Psychotria biaurita (Hutch. \& Dalziel) Verdc.

Psychotria bidentata (Benth.) Hiern

Psychotria brachyantha Hiern

$\begin{array}{cc}\text { GC } & \mathrm{mP} \\ \text { GC } & \mathrm{np} \\ \text { GC } & \mathrm{Th} \\ \text { GCW } & \mathrm{np} \\ \text { GC-SZ } & \mathrm{Ch} \\ \text { GC-SZ } & \mathrm{Ch} \\ \text { GC-SZ } & \mathrm{Th} \\ \text { GC-SZ } & \mathrm{Ch} \\ \text { SZ } & \mathrm{Th}\end{array}$

$\mathrm{GC} \quad \mathrm{np}$

GC-SZ np

$\mathrm{GC} \quad \mathrm{mp}$

$\mathrm{GC} \quad \mathrm{mp}$

GC-SZ np

$\mathrm{GC} \quad \mathrm{mp}$

GC np

$\mathrm{GC} \quad \mathrm{np}$

GC $\quad \mathrm{Ch}$

$\mathrm{GC} \quad \mathrm{mp}$

$\mathrm{GC} \quad \mathrm{mp}$

GCW mp

GCW mp

GCW $\mathrm{mP}$

GC-SZ mp

$\mathrm{SZ} \quad \mathrm{mp}$

$\mathrm{GC} \quad \mathrm{np}$

GC-SZ mp

GCW $\mathrm{mp}$

GCW mp

GC-SZ np

$\mathrm{GC} \quad \mathrm{mp}$

GCW mp

GC-SZ Ch

GC Lmp

$\mathrm{SZ} \quad \mathrm{mp}$

$\mathrm{SZ} \quad \mathrm{mp}$

GCI np

GCW np

GCW np

GCW np

GCW np

GC np 
Psychotria brachyanthoides De Wild

Psychotria dorotheae Wernham

Psychotria ealaensis De Wild

Psychotria elongatosepala (De Wild.) E.M.A.Petit

Psychotria fernandopoensis E.M.A.Petit

Psychotria gabonica Hiern

Psychotria globosa Hiern

Psychotria guineensis E.M.A.Petit

Psychotria humilis Hiern var. humilis

Psychotria humilis var. cornuta (Hiern) E.M.A.Petit

Psychotria kitsonii Hutch. \& Dalziel

Psychotria leptophylla Hiern

Psychotria limba Scott-Elliot

Psychotria linderi Hepper

Psychotria longituba A.Chev. ex De Wild

Psychotria mangenotii (Aké Assi) Verdc.

Psychotria obscura Zoll. \& Moritzi

Psychotria ombrophila (Schnell) Verdc.

Psychotria peduncularis (Salisb.) Steyerm.

Psychotria peduncularis var. guineensis (Schnell) Verdc.

Psychotria peduncularis var. hypsophila (K.Schum. \&

K.Krause) Verdc.

Psychotria peduncularis var. ivorensis (Schnell) Verdc

Psychotria peduncularis var. suaveolens (Schweinf. ex

Hiern) Verdc.

Psychotria peduncularis var. tabouensis (Schnell) Verdc.

Psychotria psychotrioides (DC.) Roberty

Psychotria rufipilis A.Chev. ex De Wild.

Psychotria sadebeckiana K.Schum

Psychotria schweinfurthii Hiern

Psychotria spathacea (Hiern) Verdc

Psychotria subglabra De Wild

Psychotria subobliqua Hiern

Psychotria umbellata Thonn

Psychotria vogeliana Benth

Psychotria yapoensis (Schnell) Verdc

Psydrax acutiflora (Hiern) Bridson

Psydrax arnoldiana (De Wild. \& T.Durand) Bridson

Psydrax horizontalis (Schumach. \& Thonn.) Bridson

Psydrax manensis (Aubrév. \& Pellegr.) Bridson.

Psydrax parviflora (Afzel.) Bridson

Psydrax schimperiana (A.Rich.) Bridson

Psydrax subcordata (DC.) Bridson

Richardia brasiliensis Gomes

$\begin{array}{cc}\text { GCI } & \text { np } \\ \text { GC } & \text { np } \\ \text { GC } & \text { Lmp } \\ \text { GC } & \text { Lmp } \\ \text { GC } & \text { np } \\ \text { GC } & \text { mp } \\ \text { GC } & \text { np } \\ \text { GC } & \text { mp } \\ \text { GC } & \text { Ch } \\ \text { GC } & \text { Ch } \\ \text { GC } & \text { np } \\ \text { GC } & \text { np } \\ \text { GCW } & \mathrm{mp} \\ \text { GCW } & \text { np } \\ \text { GCW } & n p \\ \text { GCW } & \text { Ch } \\ \text { GC-SZ } & n p \\ \text { GCW } & n p \\ \text { GC } & n p \\ \text { GC } & n p\end{array}$

$\mathrm{GC} \quad \mathrm{np}$

$\mathrm{GC} \quad \mathrm{mp}$

GC np

GCI np

GC-SZ $\quad \mathrm{mp}$

GCW np

GC np

GC-SZ np

GC np

GCW np

$\mathrm{GC} \quad \mathrm{np}$

GC np

GC-SZ np

GCW $\quad$ Ch

$\mathrm{GC} \quad \mathrm{mp}$

$\mathrm{GC} \quad \mathrm{mP}$

GC-SZ Lmp

GCW $\mathrm{mp}$

$\mathrm{GC} \quad \mathrm{mp}$

$\mathrm{SZ} \quad \mathrm{mp}$

$\mathrm{GC} \quad \mathrm{mp}$

SZ $\quad \mathrm{Ch}$ 
210 Rothmannia longiflora Salisb

211 Rothmannia munsae (Schweinf. ex Hiern) E.M.A.Petit

Rothmannia munsae subsp. megalostigma (Wernham)

$\mathrm{GC} \quad \mathrm{mp}$

GC mp

213 Rothmannia urcelliformis (Hiern) Bullock ex Robyns

$\mathrm{GC}$

mp

214 Rothmannia whitfieldii (Lindl.) Dandy

GC

$\mathrm{mp}$

215 Rutidea membranacea Hiern

GC Lmp

216 Rutidea parviflora DC

217 Rutidea rufipilis Hiern

GC-SZ

Lmp

Rutidea smithii Hiern

$\mathrm{GC}$

Lmp

GC

Lmp

219 Rytigynia argentea (Wernham) Robyns

$\mathrm{GC}$

220 Rytigynia canthioides (Benth.) Robyns

$\mathrm{GC} \quad \mathrm{mp}$

Rytigynia gracilipetiolata (De Wild.) Robyns

$\mathrm{GC}$

mp

Rytigynia senegalensis Blume

GC-SZ

$\mathrm{mp}$

Rytigynia umbellulata (Hiern) Robyns

$\mathrm{GC}$

$\mathrm{mp}$

Sabicea brevipes Wernham

GC-SZ

mp

Sabicea calycina Benth

Sabicea cordata Hutch. \& Dalziel

GC

Lmp

Sabicea ferruginea (G.Don) Benth

GCW

Lnp

Sabicea harleyae Hepper

GCW

Lnp

GCW

Lmp

229 Sabicea rosea Hoyle

$\mathrm{GC}$

Lmp

$230 \quad$ Sabicea venosa Benth

GC

Lmp

$231 \quad$ Sabicea vogelii Benth.

232 Sacosperma paniculatum (Benth.) G.Taylor

GCW Lmp

GC Lmp

233 Sarcocephalus latifolius (Sm.) E.A.Bruce

$\mathrm{SZ} \quad \mathrm{mp}$

234 Schizocolea linderi (Hutch. \& Dalziel) Bremek.

GCW np

235 Schumanniophyton problematicum (A.Chev.) Aubrév.

GCW $\quad \mathrm{mp}$

Sericanthe chevalieri (K.Krause) Robbr

SZ

mp

Sericanthe toupetou (Aubrév. \& Pellegr.) Robbr

GCI

GC

GCW

$\mathrm{mp}$

Sherbournia bignoniiflora (Welw.) Hua

Lmp

239 Sherbournia calycina (G.Don) Hua

$\mathrm{GC}$

Lmp

Spermacoce alata Aubl.

Spermacoce exilis (L.O.Williams) C.D.Adams ex

Spermacoce filifolia (Schumach. \& Thonn.) J.-P.Lebrun \&

244 Spermacoce hepperiana Verdc.

GC-SZ Th

245 Spermacoce intricans (Hepper) H.M.Burkill

GCW

Spermacoce ivorensis Govaerts

GCW

GC-SZ

247 Spermacoce octodon (Hepper) Hakki

$\mathrm{GC}$ 
250 Spermacoce quadrisulcata (Bremek.) Verdc.

251 Spermacoce radiata (DC.) Hiern

252 Spermacoce ruelliae DC.

GC-SZ

253 Spermacoce stachydea DC.

GC-SZ Th

254 Spermacoce verticillata $\mathrm{L}$.

GC-SZ Th

255 Stipularia africana P.Beauv

GC-SZ np

256 Tarenna bipindensis (K.Schum.) Bremek.

$\mathrm{GC}$

np

257 Tarenna conferta (Benth.) Hiern

GC

GC-SZ

Lmp

258 Tarenna eketensis Wernham

259 Tarenna fuscoflava (K.Schum.) S.Moore

GC

$\mathrm{mp}$

260 Tarenna gracilis (Stapf) Keay

GC

GCW

Lmp

261 Tarenna nitidula (Benth.) Hiern

GCW

Lmp

262 Tarenna pavettoides (Harv.) Sim

$\mathrm{GC}$

$\mathrm{mp}$

263 Tarenna thomasii Hutch. \& Dalziel

GC

$\mathrm{mp}$

264 Tarenna vignei Hutch. \& Dalziel

$\mathrm{GCW}$

$\mathrm{mp}$

265 Tricalysia biafrana Hiern

266 Tricalysia coriacea (Benth.) Hiern

$\mathrm{GC} \quad \mathrm{mp}$

267 Tricalysia faranahensis Aubrév. \& Pellegr.

GC

GCW $\quad \mathrm{mp}$

268 Tricalysia okelensis Hiern

GC-SZ mp

269 Tricalysia pallens Hiern

$\mathrm{GC}$

mp

270 Tricalysia reticulata (Benth.) Hiern

GCW

np

271 Trichostachys aurea Hiern

$\mathrm{GC}$

$\mathrm{Ch}$

272 Uncaria africana G.Don

GC

LmP

273 Uncaria talbotii Wernham

$274 \quad$ Vangueria agrestis (Schweinf. ex Hiern) Lantz

GC

LmP

275 Vangueriella discolor (Benth.) Verdc.

SZ np

276 Vangueriella glabrescens (Robyns) Verdc.

GCW

mp

277 Vangueriella nigerica (Robyns) Verdc.

GC LmP

278 Vangueriella orthacantha (Mildbr.) Bridson \& Verdc.

GC-SZ $\quad \mathrm{mp}$

279 Vangueriella spinosa (Schumach. \& Thonn.) Verdc.

$\mathrm{GC} \quad \mathrm{mp}$

280 Vangueriella vanguerioides (Hiern) Verdc.

GC-SZ $\quad \mathrm{mp}$

281 Virectaria multiflora (Sm.) Bremek.

GCW $\quad \mathrm{mp}$

282 Virectaria procumbens (Sm.) Bremek.

GC-SZ

Th

Type chorologique: GC : Guinéo-Congolais; GC-SZ: Guinéo-Congolaises-Soudano-Zambéziennes; SZ : SoudanoZambéziennes; GCW : espèces endémiques du bloc forestier à l'ouest du Togo; GCI : espèces endémiques de la Côte d'Ivoire. Type biologique : mp : microphanérophytes; $\mathrm{np}$ : nanophanérophytes ; Lmp : microphanérophytes lianescentes ; LmP : mésophanérophytes lianescentes; MP : mégaphanérophytes; $\mathrm{Ch}$ : Chaméphytes; $\mathrm{Th}$ : thérophytes; $\mathrm{mP}$ : mésophanérophytes ; Lnp : nanophanérophytes lianescentes; Lmp (mp): microphanérophytes lianescentes ; Hyp : hémicryptophytes pyrophytique. 


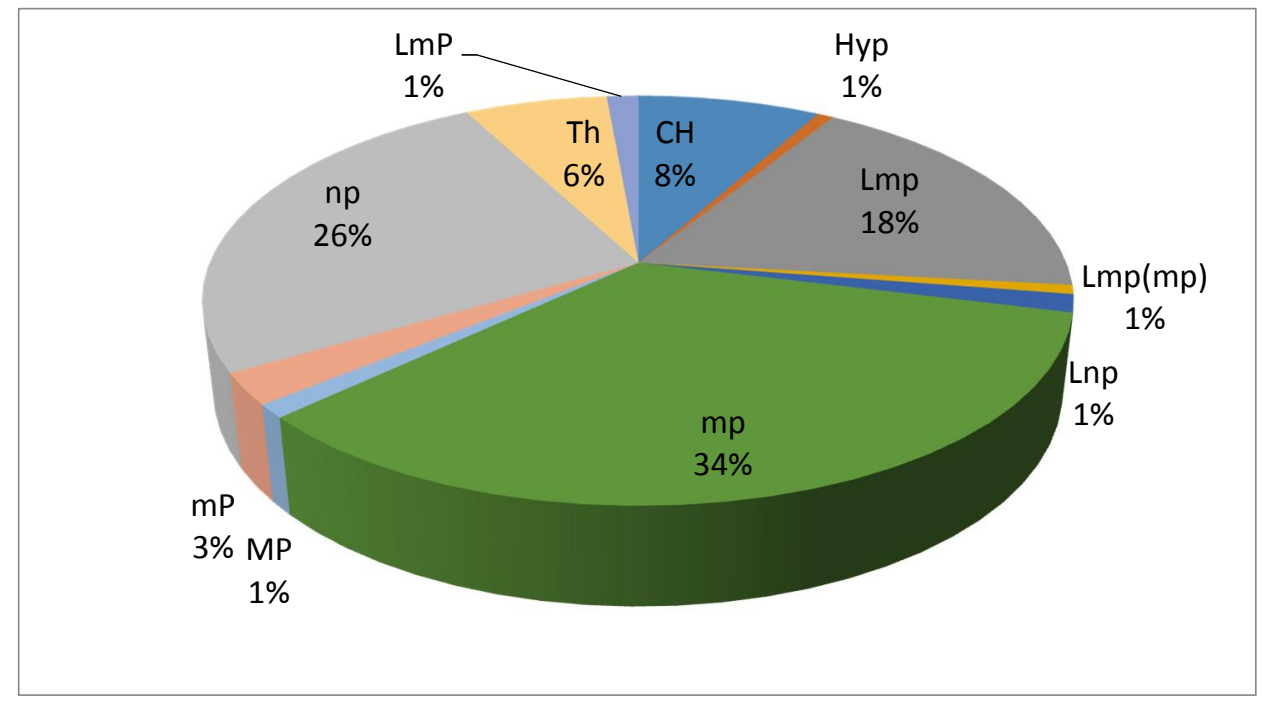

Figure 4: Type biologique des espèces de la famille des Rubiaceae de la base de données SIG IVOIRE (mp : microphanérophytes; $\mathrm{np}$ : nanophanérophytes ; Lmp : microphanérophytes lianescentes; LmP : mésophanérophytes lianescentes; MP : mégaphanérophytes; $\mathrm{Ch}$ : Chaméphytes ; Th : thérophytes ; $\mathrm{mP}$ : mésophanérophytes ; Lnp : nanophanérophytes lianescentes ; Lmp (mp) : microphanérophytes lianescentes ; Hyp : hémicryptophytes pyrophytique).

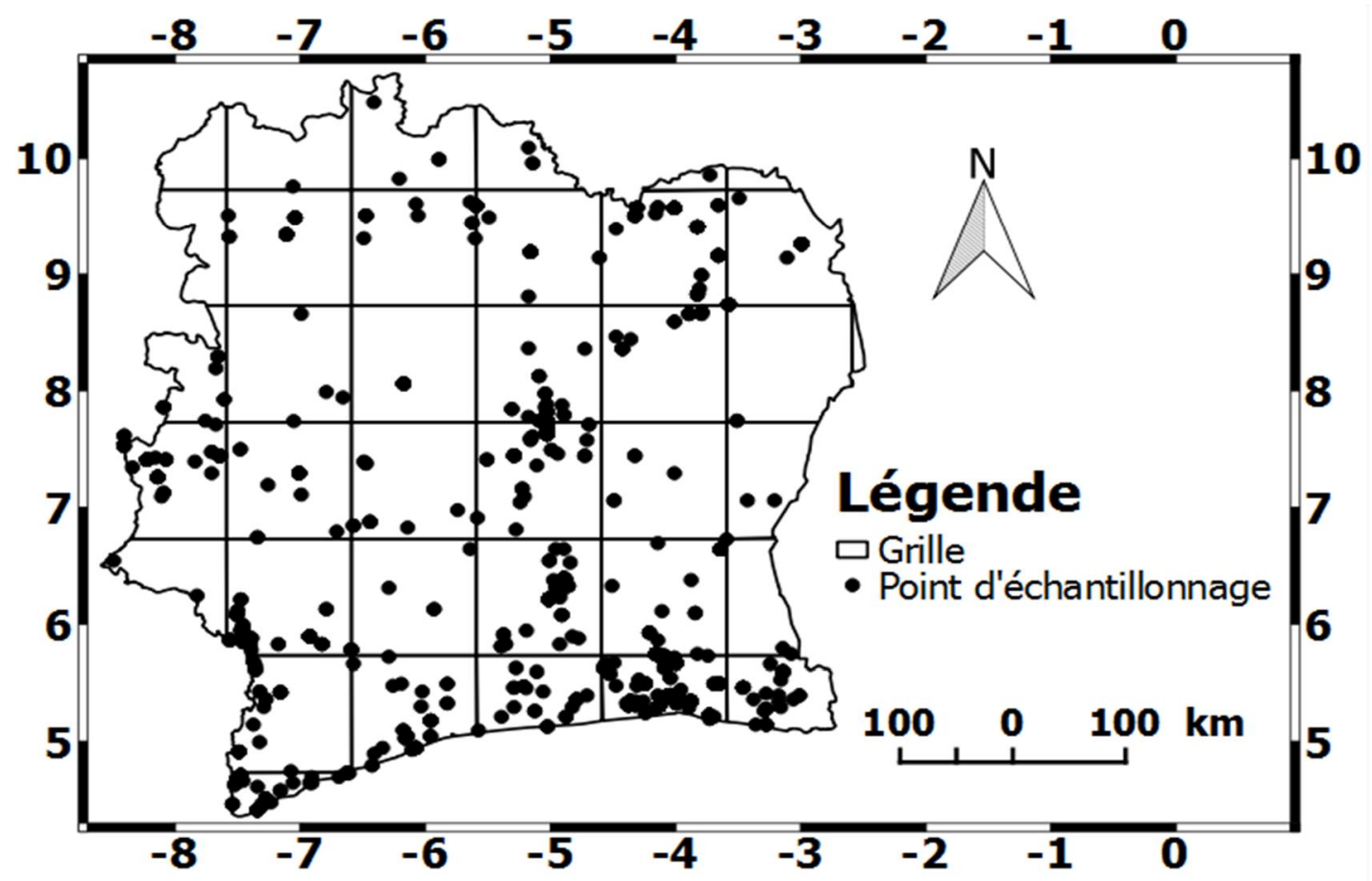

Figure 5: Carte de distribution des échantillons de Rubiaceae de la base SIG IVOIRE. 


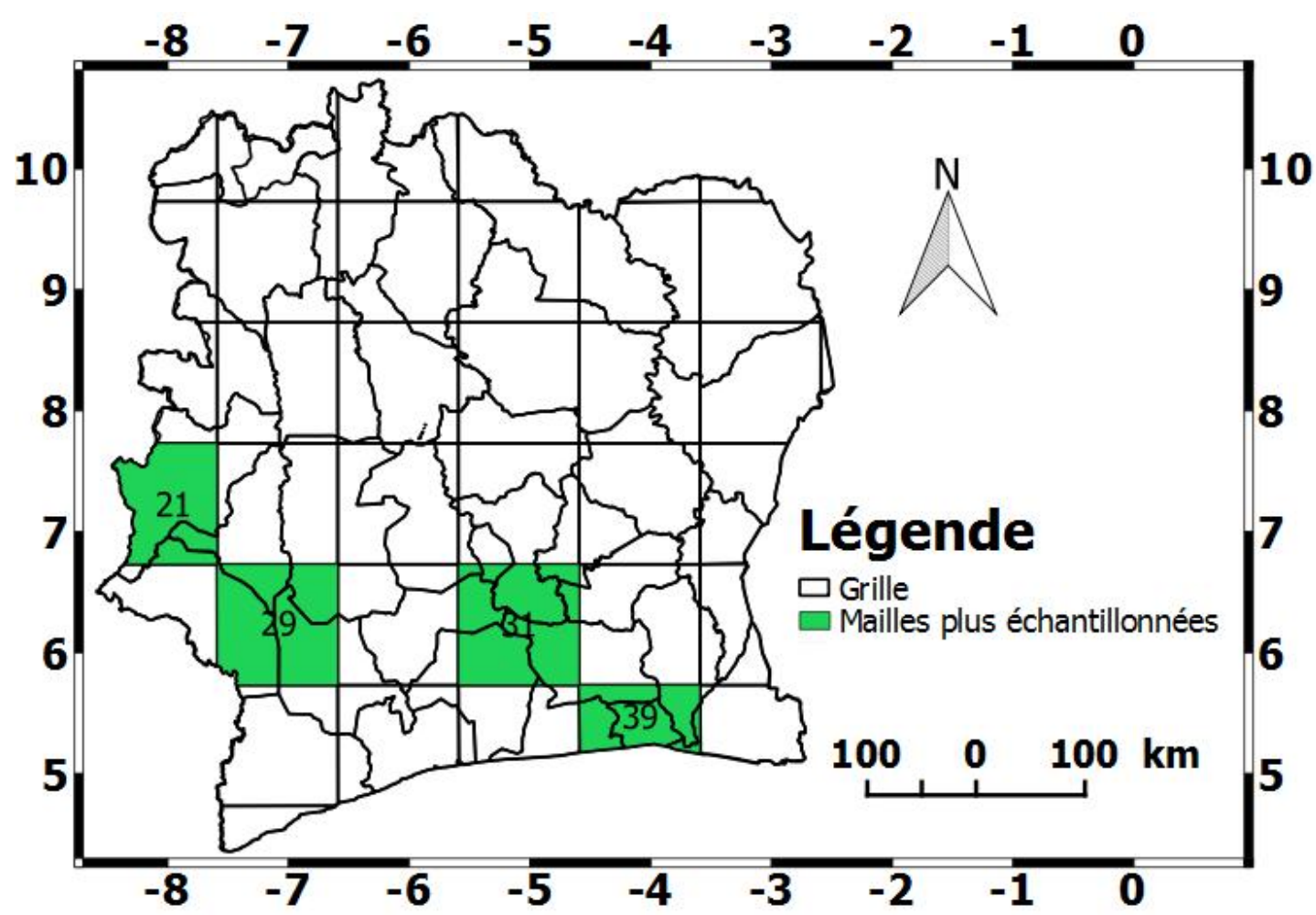

Figure 6: Carte montrant les zones floristiquement connues.

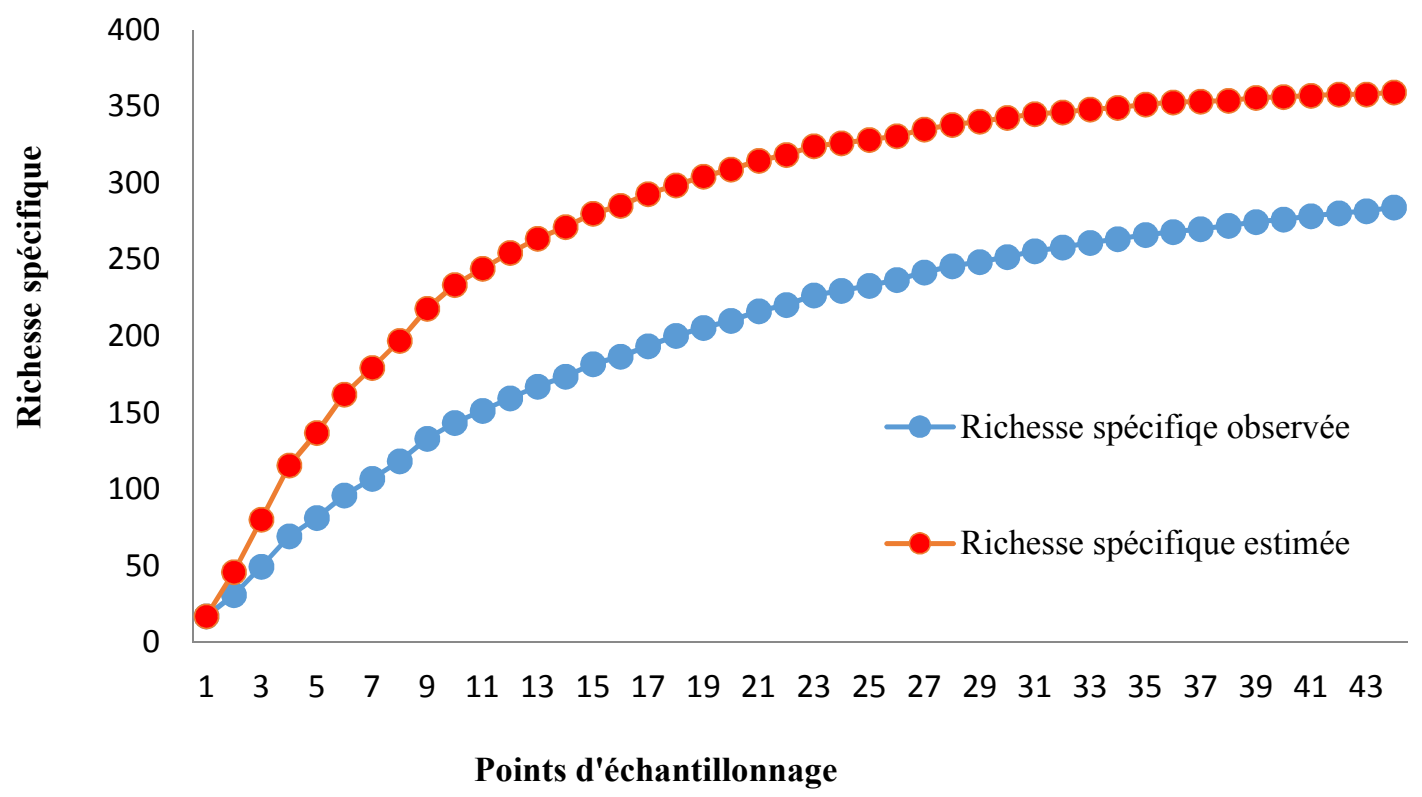

Figure 7: Courbe d'accumulation des espèces dans les différentes mailles. 


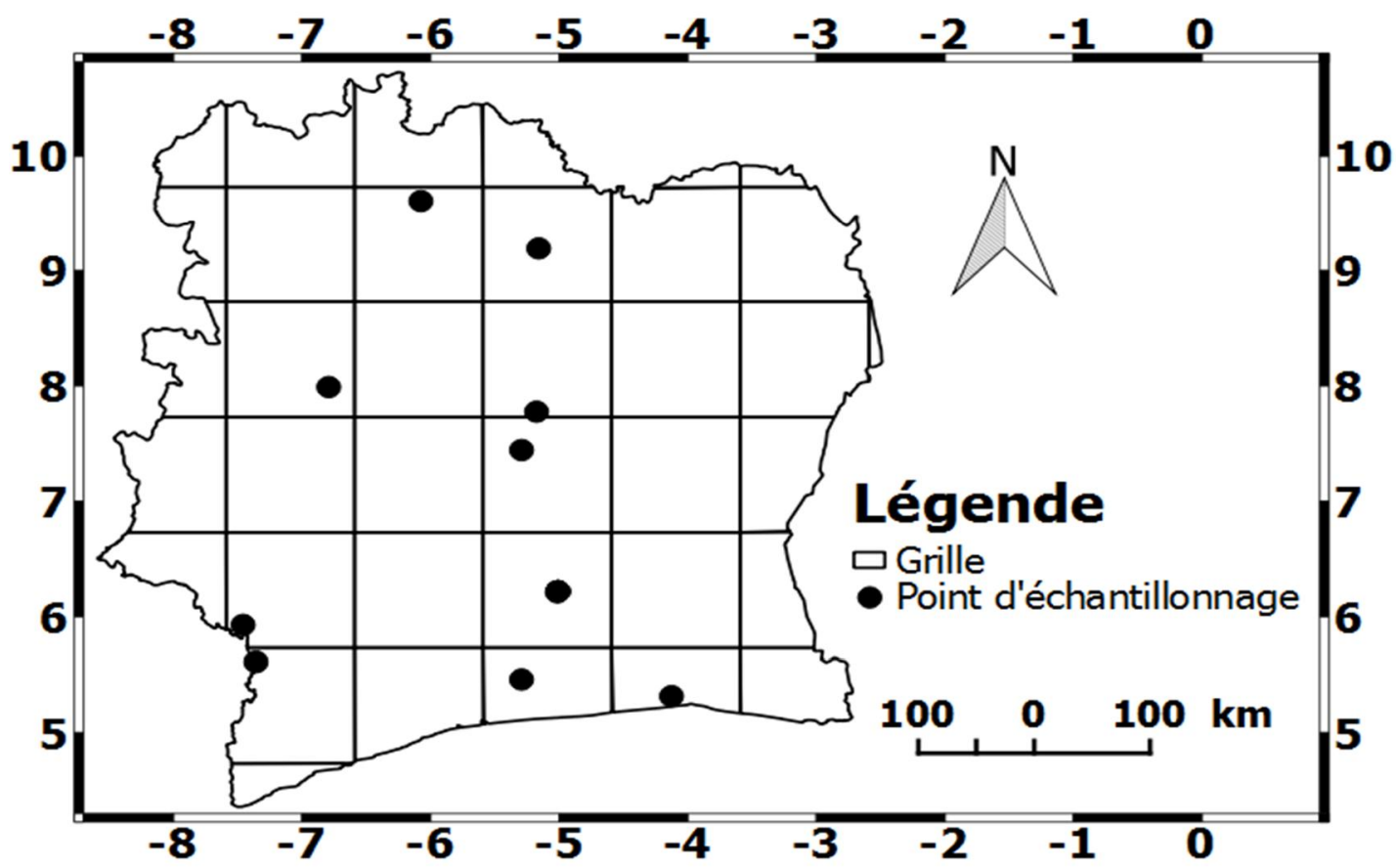

Figure 8: Carte de distribution de Rothmannia longiflora Salisb avec 15 échantillons, récoltés dans 10 mailles.

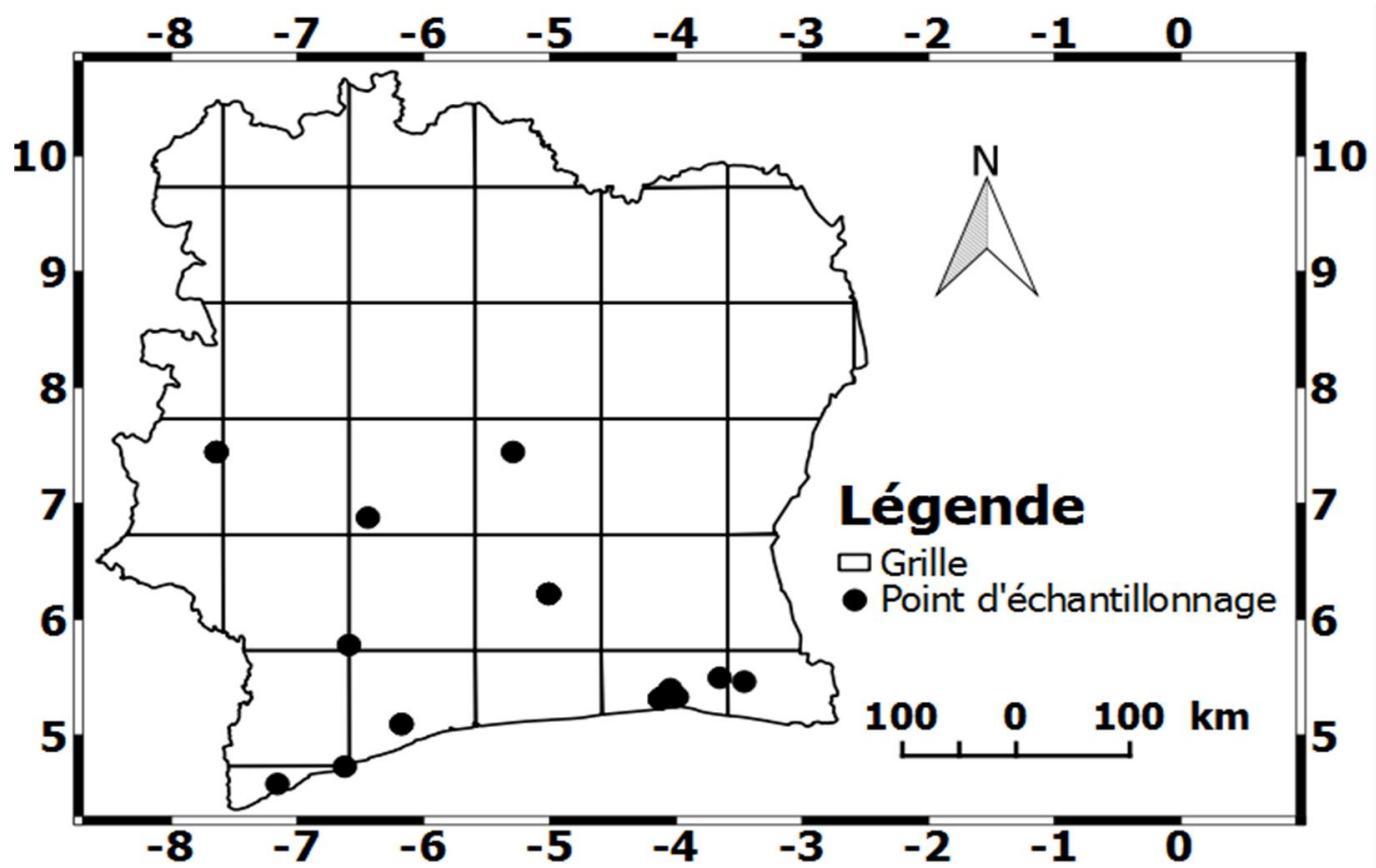

Figure 9: Carte de distribution de Chassalia kolly (Schumach.) Hepper avec 23 échantillons, récoltés dans 9 mailles. 


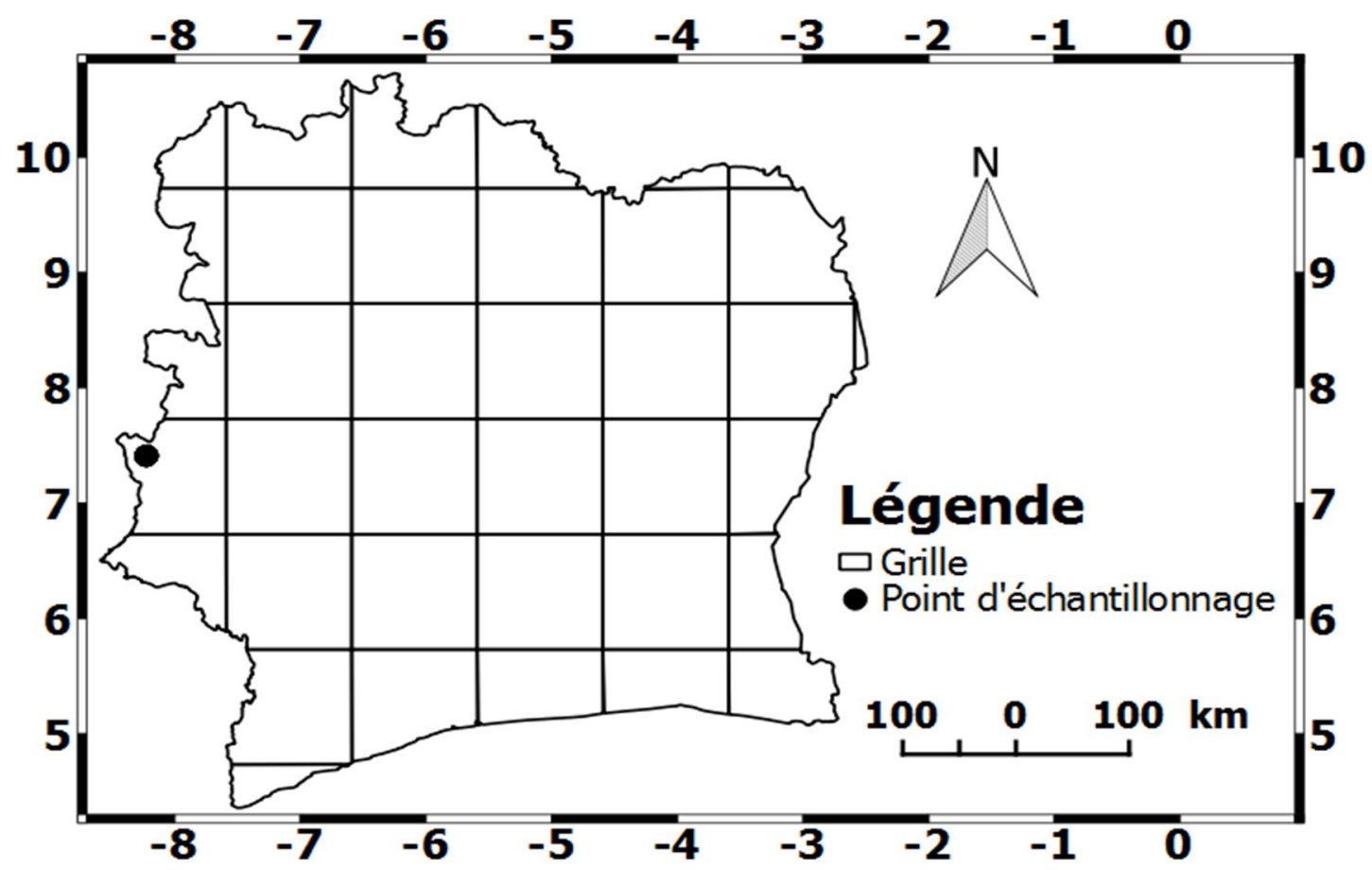

Figure 10: Carte de distribution de Psychotria limba Scott-Elliot avec 4 échantillons récoltés dans une seule maille.

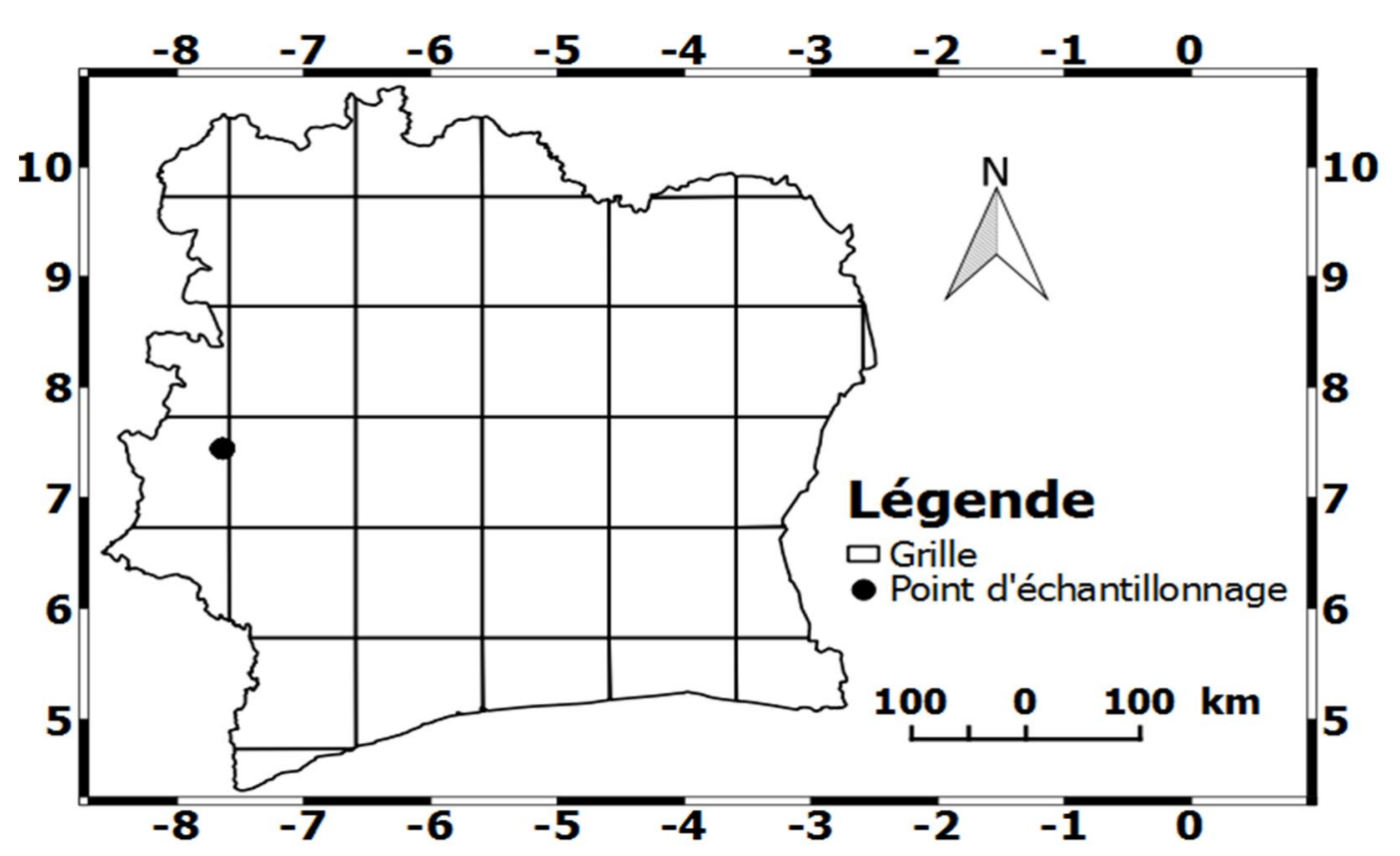

Figure 11: Carte de distribution de Tricalysia reticulata (Benth.) Hiern avec 7 échantillons, récoltés dans une seule maille. 


\section{DISCUSSION}

Les espèces de la famille des Rubiaceae de la flore ivoirienne, sont généralement de type Guinéo-Congolaise (GC), cette structure chorologique telle qu'elle apparaît dans nos résultats est très comparable à celle des formations de forêts denses humides sempervirentes (Kouamé et al., 2010). Selon Doumenge et al. (2003), ces forêts renferment une flore très riche avec un taux d'endémisme élevé. Une grande partie des récoltes des espèces de la famille des Rubiaceae est faite dans le secteur ombrophile. Cette répartition en général, est majoritairement dans la zone forêt dense humide sempervirente, dans les forêts denses humides semi décidue et les savanes guinéennes et minoritairement dans la savane soudanaise. Ces résultats sont confirmés par, Denguéadhé et al. (2006) qui stipulent que les forêts sempervirentes sont dominées par les Rubiaceae. Quant aux types biologiques, les phanérophytes dominent, néanmoins, les lianes occupent une place de choix comme dans tout milieu forestier tropical. La densité de récolte dans les différentes mailles est importante en ce sens qu'elle nous renseigne sur le niveau de prospection de chaque maille. Cependant, la superposition de la carte des mailles avec celle des régions montrent que certaines régions de la Côte d'Ivoire sont floristiquement connues vis-à-vis des Rubiaceae. Ce sont la région de l'AgnébyTiassa, du district d'Abidjan, du Tompki, des Grands Ponts, du Belier et du Cavally. Ces constats sont conformes à ceux de Koffi et al. (2015). En effet, les régions les mieux connues floristiquement sont celles situées le long de la côte, les zones environnantes des centres d'activités et des institutions de recherche et des universités, des forêts classées, des parcs et réserves naturelles (le Parc National du Banco, la forêt classée de Yapo et la réserve de Lamto). En dehors de ces régions, de grands espaces très diversifiés en végétation naturelle ne sont pas explorés ou ne le sont que très partiellement (Koffi, 2008). Selon Hepper, cité par Koffi (2005), en Afrique, il y a des régions bien connues, des régions moyennement connues et des régions mal connues. Cette étude fournit des analyses détaillées et dresse une carte de l'effort d'échantillonnage afin de donner des informations phytogéographiques. Ces informations devraient guider de nouvelles enquêtes botaniques en Côte d' Ivoire. Par ailleurs, les espèces récoltées dans plus d'une maille sur l'ensemble de la zone d'étude sont des espèces avec une large amplitude écologique. Elles sont capables de s'adapter aux facteurs édaphiques et abiotiques divergents. C'est le cas de Chassalia kolly (Schumach.) Hepper et Keetia venosa (Oliv.) Bridson etc. Ces espèces à large distribution spatiale ont une facilité de reproduction dans différents types de climats, de végétations et de sols (Adomou et al., 2012). Par contre, celles récoltées dans seulement une maille bien précise sont des espèces avec vraisemblablement une amplitude écologique plus restreinte. C'est le cas de Tricalysia reticulata (Benth.) Hiern, Geophila obvallata Didr. etc. Cela pourrait être dû aux variables environnementales et selon Boko et al. (2007), beaucoup d'espèces n'ont pu suivre les changements climatiques relativement rapides, ce qui pourrait expliquer la présence de certaines espèces dans des zones restreintes. D'autres encore, bien qu'étant abondamment récoltées dans une zone, ont quelques spécimens dans les zones voisines. Ces dernières peuvent être qualifiées d'espèces de transition (Koffi et al., 2008).

Dans le cadre de la conservation, la valeur indicatrice des Rubiaceae n'est pas constante dans toutes les mailles en raison de la variation $\mathrm{du}$ nombre de représentation par espèce. L'utilisation d'espèces avec un nombre restreint d'échantillons pour caractériser les zones de haute importance donc devraient être aperçue avec prudence. Les valeurs attachées à la biodiversité peuvent varier au niveau 
local, national et international. Dans la présente étude, la valeur pour la conservation de la biodiversité est déterminée à travers la présence de plusieurs espèces à statut particulier: espèces endémiques, rares et menacées d'extinction (Jongkind, 2004 ; UICN, 2012). Ces espèces sont majoritairement localisées dans la zone d'endémisme (hotspot) de la Côte d'Ivoire.

\section{Conclusion}

Cette étude est une contribution à la protection et la conservation de la biodiversité de la Côte d'Ivoire à travers la famille des Rubiaceae. $\mathrm{Au}$ terme de cette étude, nous avons analysé la diversité et la distribution spatiale des espèces de Rubiaceae récoltées en Côte d'Ivoire. Les espèces de Rubiaceae sont généralement de types Guinéo-Congolaise. Cependant l'analyse des résultats révèlent que la carte de distribution de tous les échantillons montre une exploration irrégulière sur le territoire ivoirien. Ainsi, l'on détermine des zones bien connues floristiquement et des zones mal connues en Côte d' Ivoire. La cartographie des espèces indique qu'il a des espèces avec une distribution large par contre d'autres espèces sont confinés dans des endroits spécifiques. Les espèces à distribution restreinte et les espèces endémiques répertoriées mise en évidence dans cette étude méritent une attention particulière et seraient prioritaires pour la conservation.

\section{CONFLITS D'INTERETS}

Les auteurs déclarent qu'ils n'ont aucun conflit d'intérêts.

\section{CONTRIBUTIONS DES AUTEURS}

FNT a réalisé à la redaction du manuscrit. KJK a contribué à la réalisation des cartes de distribution. AFK a contribué à l'analyse de la diversité. BA a facilité l'accès à la base de données. MK et JB ont contribué par leur lecture à l'améliration et à la validation du manuscrit.

\section{REMERCIEMENTS}

Les auteurs remercient le Centre Suisse de Recherche Scientifique (CSRS) qui a mis à notre disposition la base de données SIG IVOIRE. Ils remercient vivement Monsieur Cyrille Chatellain et ses collaborateurs qui sont les auteurs de cette base de données.

\section{REFERENCES}

Adomou AC, Yedomonhan H, Djossa B, Legba SI, Oumorou M, Akoegninou A. 2012. Etude ethnobotanique des plantes médicinales vendues dans le marché d'Abomey Calavi au Bénin. Int. J. Biol. Chem. Sci., 6(2): 745-772. DOI: http://dx.doi.org/10.4314/ijbcs. v6i2.18.

Aké Assi L. 2001. Flore de la Côte d'Ivoire: catalogue systématique, biogéographique et écologique. I. Boissiera, 57, 396. DOI : http://bibliotheques.mnhn.fr/ medias/detailstatic.aspx?INSTANCE $=\mathrm{ex}$ ploitation\&RSC_BASE $=$ HORIZON\&R SC_DOCID $=312431$

Aké Assi L. 2002. Flore de la Côte d'Ivoire: catalogue systématique, biogéographique et écologique. II. Boissiera, 58, 441 p.

Assoumou MR, Mezui, Mate JP, Mweru, Tchouamo IR. 2012. Impact du développement des palmeraies familiales sur la déforestation et dans les ménages au Cameroun. Int. J. Biol. Chem. Sci. 6(6): 3017-3026. http://indexmedicus. afro.who.int

Boko M, Niang I, Nyong A, Vogel C, Githeko A, Medany M,Osman-Elasha B, Tabo R, Yanda P. 2007. Africa. Climate Change 2007: Impacts, Adaptation and Vulnerability. Contribution of Working Group II to the Fourth Assessment Report of the Intergovernmental Panel on Climate Change, Parry ML, Canziani OF, Palutikof JP, van der Linden PJ, 
Hanson CE (eds). Cambridge University Press: Cambridge UK. pp. 433 - 467.

Brou YT, Oswald Y, Bigot S, Servat E. 2005. Risques de déforestation dans le domaine permanent de l'Etat en Côte d'Ivoire: quel avenir pour ses derniers massifs forestiers? TELA 2004004, Revue de Télédétection de l'AUF, 5(1-2-3): 17-33. DOI : https://www.researchgate.net/ /264195874

Burnham KP, Overton PS. 1978. Estimation of the size of closed population when capture probabilities vary among animals. Biometrika, 65: 625-633. DOI: $10.2307 / 2335915$

Chatelain C, Dao H, Gautier L, Spichiger R. 2004. Forest cover changes in Côte d'Ivoire and Upper Guinea. In Biodiversity of West African Forests, An Ecological Atlas of Woody Plant Species ( $1^{\text {ere }}$ edition). CABI Publ. 15-32.

Chatelain C, Gautier L, Spichiger R. 2001. Application du SIG Ivoire à la distribution potentielle des espèces en fonction des facteurs écologiques. Proceedings of the XVI AETFAT Congress. Meise, 71: 313-326.

Crisp MD, Laffan S, Linder HP, Monro A. 2001. Endemism in the Australian flora. Journal of Biogeography, 28: 183-198. http://biology-assets.anu.edu.au/ hosted_sites/Crisp/pdfs/Crisp2001_ende mism.pdf

Davis AP, Figueiredo E. 2007. A checklist of the Rubiaceae (coffee family) of Bioko and Annobon (Equatorial Guinea, Gulf of Guinea). System. Biod. Journal, 5(2): 159-186.

Davis AP, Govaerts R, Diane M, Bridson M, Ruhsam M, Moat J, Brummitt A. 2009. A global assessment of distribution, diversity, endemism and taxonomic effort in the Rubiaceae. An. Miss. Bot. Garden, 96(1): 1-11. DOI: $10.3417 / 2006205$
Denguéadhé KTS, Decocq G, Adou Yao CY, Blom EC, Van Rompaey RSAR. 2006. Plant species diversity in the southern part of the Taï National Parc (Côte d'Ivoire). Biodiversity and Conservation, 15: 2140-2142. DOI: 10.1007/s10531004-6686-1.

Doumenge C, Ndinga A, Fomete NT, Tchanou Z, Micha OV, Ona NN, Bourobou BH, Ngoye A. 2003. Conservation de la biodiversité forestière en Afrique centrale atlantique. II. Identification d'un réseau de sites critiques. Bois et Forêts des Tropiques, 276: $43-58$.

Eddouks M, Ouahidi ML, Farid O, Moufid A, Khalidi A, Lemhadri A. 2007. L'utilisation des plantes médicinales dans le traitement du diabète au Maroc. Phytothérapie, 5: 194-203. DOI: 10.1007/s10298-007-0252-4.

Jongkind CCH. 2004. Checklist of Upper Guinea forest species. In Biodiversity of West African Forests: An Ecological Atlas of Woody Plant Species. Poorter LF, Bongers F, Kouamé FN, Hawthorne WD (eds). Cabi Publishing: London; 447- 477 .

Koffi KJ. 2005. Analyse des structures spatiales des données de distribution phytogéographique en Afrique centrale. Mémoire de DEA, Université libre de Bruxelles, Belgique, $190 \mathrm{p}$.

Koffi KJ. 2008. Analyse des structures spatiales des données de distribution phytogéographique des Acanthaceae en Afrique centrale. Thèse de Doctorat. Université Libre de Bruxelles, Faculté des sciences, Ecole Interfacultaire de Bioingenieurs, Service d'Ecologie du paysage et Systèmes de production végétale, $240 \mathrm{p}$.

Koffi KJ, Champluvier D, Danho FRN, De Cannière C, Traoré D, Lejoly J, Robbrecht E, Bogaert J. 2008. Analyse de la distribution spatiale des 
Acanthaceae en Afrique Centrale et comparaison avec les théories phytogéographiques de Robyns, White et Ndjele. Sciences et Nature, 5(2) : 101 110. https://www.ajol.info/index.php/ scinat/article/view/42156/9284.

Koffi KJ, Kouassi AF, Adou Yao. CY, Bakayoko A, Ipou JI, Bogaert J. 2015. The present state of botanical knowledge in Côte d'Ivoire. Biodiversity Informatics, 10: 56-64. https://journals.ku.edu/jbi/article/view/50 07/4495.

Koné M, Kouadio YL, Neuba DFR, Malan DF, Coulibaly L. 2014. Evolution de la couverture forestière de la Côte d'Ivoire des années 1960 au début du $21^{\mathrm{e}}$ siècle. International Journal of Innovation and Applied Studies, 7(2): 782- 794. http://www.ijias.issr-journals.org/

Kouamé NF, Zoro Bi IA. 2010. Nouveau découpage de la zone de forêt dense humide de la Côte d'Ivoire. Sciences et Nature, $\quad 7(2): \quad 177 \quad$ - 194. https://www.ajol.info/index.php/scinat/ar ticle/view/59962/48232.

Kouassi AF, Koffi KJ, Ipou IJ. 2015. Etude agrostologique et socioéconomique des exploitations fourragères. Edition Universitaire Européenne, 204 p.

Lachenaud O. 2013. Le genre Psychotria (Rubiaceae) en Afrique occidentale et centrale : taxonomie, phylogénie et biogéographie/The genus Psychotria (Rubiaceae) in West and Central Africa: taxonomy, phylogeny and biogeography. Thèse de Doctorat, Faculté des Sciences, Université Libre de Bruxelles/Belgique, $1121 \mathrm{p}$.

Lachenaud O, Droissart V, Dessein S, Stévart T, Simo M, Lemaire B, Taedoumg H, Sonké B. 2013. New records for the flora of Cameroon, including a new species of Psychotria (Rubiaceae) and range extensions for some rare species. $\mathrm{Pl}$.
Ecol. Evol., 146(1/3): 121-133. doi:10.5091/plecevo.2013.632.

Lebri M, Bahi C, Fofié NBY, Gnahoue G, Lagou SM, Achibat H, Yapi A, Zirihi GN, Coulibaly A, Hafid A, Khouili M. 2015. Analyse phytochimique et évaluation de la toxicité aiguë par voie orale chez des rats de l'extrait total aqueux des feuilles de Abrus precatorius Linn (Fabaceae). Int. J. Biol. Chem. Sci., 9(3): 1470-1476. DOI : http://dx.doi.org/10.4314/ijbcs.v9i3.29.

Mayaux P, Bartholomé E, Massart M, Van Cutsem C, Cabral A, Nonguierma A, Diallo O, Pretorius C, Thompson M, Cherlet M, Pekel J-F, Defourny $\mathrm{P}$, Vasconcelos M, Di Gregorio A, Fritz S, De Grandi G, Elvidge C, Vogt P, Belward A. 2003. Carte de l'occupation du sol de l'Afrique, EUR 20665 EN, European Commission, Joint Research Center, $56 \mathrm{p}$.

Menziès A. 2000. Structure et composition floristique de la forêt de la zone Ouest du Parc National de Taï (Côte d'Ivoire). Mémoire de Master, Université de Genève, 194 p.

Pielou EC. 1975. Ecological Diversity. John Wiley \& Sons: New York; 165.

Shannon CE, Weaver W. 1949. The Mathematical Theory of Communication. University of Illinois Press: Urbana. 125 p.

UICN. 2012. IUCN Red List of Threatened Species, Version 2012, 2, [En ligne], URL: http://www.iucnredlist.org, Consulté le 7 janvier 2013.

Yapi AB, Kassi NJ, Fofié NBY, Zirihi GN. 2015. Etude ethnobotanique des Asteraceae médicinales vendues sur les marches du district autonome d'Abidjan (Côte d'Ivoire). Int. J. Biol. Chem. Sci., 9(6): 2633-2647. http://indexmedicus. afro.who.int. 